\title{
A Simple Method for Computing Singular or Nearly Singular Integrals on Closed Surfaces
}

\author{
J. Thomas Beale ${ }^{1, *}$, Wenjun Ying ${ }^{2}$, and Jason R. Wilson ${ }^{3}$ \\ ${ }^{1}$ Department of Mathematics, Duke University, Durham, NC 27708-0320, USA. \\ 2 Department of Mathematics, MOE-LSC and Institute of Natural Sciences, \\ Shanghai Jiao Tong University, Minhang, Shanghai 200240, P. R. China. \\ ${ }^{3}$ Mathematics Department, Virginia Tech, Blacksburg, VA 24061-0123, USA.
}

\begin{abstract}
We present a simple, accurate method for computing singular or nearly singular integrals on a smooth, closed surface, such as layer potentials for harmonic functions evaluated at points on or near the surface. The integral is computed with a regularized kernel and corrections are added for regularization and discretization, which are found from analysis near the singular point. The surface integrals are computed from a new quadrature rule using surface points which project onto grid points in coordinate planes. The method does not require coordinate charts on the surface or special treatment of the singularity other than the corrections. The accuracy is about $O\left(h^{3}\right)$, where $h$ is the spacing in the background grid, uniformly with respect to the point of evaluation, on or near the surface. Improved accuracy is obtained for points on the surface. The treecode of Duan and Krasny for Ewald summation is used to perform sums. Numerical examples are presented with a variety of surfaces.
\end{abstract}

AMS subject classifications: 65R20, 65D30, 31B10, 35J08

Key words: boundary integral method, layer potential, nearly singular integrals, Laplace equation, surface integral, implicit surface

\section{Introduction}

We present a simple, accurate method for computing singular or nearly singular integrals defined on a smooth, closed surface in three-space. This method can be used to evaluate single or double layer potentials for harmonic functions, or the velocity and pressure in Stokes flow due to forces on a surface. The point of evaluation could be on or near the surface. To evaluate the integral, the kernel is first replaced by a regularized version. A preliminary value is found using a new quadrature rule for surface integrals which has the advantage that it does not require coordinate systems or a triangulation on the

*Corresponding author. Email addresses: beale@math.duke.edu (J. T. Beale), wying@sjtu.edu.cn (W. Ying), jasonwil@math.vt.edu (J. R. Wilson) 
surface. Instead we sum values of the integrand over quadrature points which project onto grid points in coordinate planes, in a way that would be high order accurate if the integrand were smooth. Corrections for the regularization and discretization are then added to achieve higher accuracy. These corrections are given by explicit formulas derived using asymptotic analysis near the singularity as in [4]. The resulting value of the integral has $O\left(h^{p}\right)$ accuracy for $p<3$, uniformly for points of evaluation near the surface, where $h$ is the grid spacing in $\mathbb{R}^{3}$. For points on the surface, the accuracy is significantly improved by using a special regularization; see Section 3.3. For efficient summation we use the treecode algorithm of Duan and Krasny [11] designed for kernels with Gaussian regularization. The method presented here could be used, for example, to find values of the potential at grid points in $\mathbb{R}^{3}$ close to the surface. It should be applicable to computations with moving surfaces for which good accuracy is needed without extensive work to represent the surface at each time step. Other kernels could be treated by the same approach, and the more accurate version of the method for computing values on the surface could be used for a variety of problems which can be formulated as integral equations.

The present approach is an improvement and extension of the grid-based boundary integral method of [4]. In the earlier work the integral was replaced by sums in coordinate charts using a partition of unity. The need for explicit coordinate systems requires knowledge of the surface that might be difficult to obtain for a moving surface. Furthermore, if the coordinate system is too distorted, the accuracy will be poor because the discretization error will fail to be controlled by the regularization. Here we avoid these disadvantages by using a more direct rule for computing surface integrals, which was introduced for smooth integrands in [30]. This quadrature rule uses projections on coordinate planes rather than coordinate charts. Given a rectangular grid in $\mathbb{R}^{3}$, quadrature points are chosen as those points on the surface which project onto grid points in the coordinate planes, for which the normal to the surface has direction away from the plane. Weights for the quadrature points are found from a partition of unity on the unit sphere, applied to the normal vector at the point. The weight functions on the sphere are chosen universally, and do not depend on the particular surface. The resulting quadrature rule for surface integrals has high order accuracy, as allowed by the smoothness of the integrand and the surface. In effect, the method uses the existence of coordinate patches without having to refer to them explicitly. The quadrature points can be found efficiently if, for example, the surface is given analytically or numerically as the level set of a function. Examples in [30] with smooth integrands illustrate the accuracy of this method with a variety of surfaces, including ones of large genus.

For a variety of problems in partial differential equations, solutions can be written as integrals over surfaces, using a known fundamental solution. These include harmonic functions, electromagnetic waves, and viscous fluid flow modeled by the Stokes equations. The specific representation makes this formulation an attractive approach for numerical methods. Often an integral equation on the surface must be solved, and much attention has focused on such problems. For the most familiar case, an integral equation for the Dirichlet problem for the Laplacian, using the double layer potential, it was 
proved in [4] that the discrete version of the integral equation using the present method has a unique solution and that it converges to the exact solution under grid refinement. The evaluation of the integral at points near the surface, in contrast to evaluation on the surface, generally requires extra care and can also be important in applications. If values of a potential are needed at grid points in the computational domain, we can first compute values at points near the surface directly as nearly singular integrals. Once this is done, values at other grid points can be found in a cheap way by inverting a discrete Laplacian, as in [20]. In this paper the values obtained at the irregular grid points near the surface using integration have accuracy about $O\left(h^{3}\right)$, while those found at the regular points are about $O\left(h^{2}\right)$ accurate; more accurate values could be found with more work.

The most widely used numerical technique for integral formulations is the boundary element method (e.g. [1, 14, 24, 25]); the boundary is triangulated and matrix elements are computed for the integral operator on the surface using special quadrature rules. This method is especially useful for electromagnetic problems in which the surface does not evolve and may have corners and edges. Direct quadrature based on triangulations is also used, and can be accurate in the (exactly) singular case for smooth surfaces. Such methods have long been used for modeling in chemistry and biology [23, 24, 36]. Some methods use corrected quadrature weights [19] or analytical evaluation of the singular part, treating the remainder in a standard way [16, 30]. A careful direct quadrature (or Nyström) method, introduced in [7] for electromagnetics, and further developed in [32], uses a partition of unity to reduce the integral to coordinate patches; a special patch in polar coordinates is used near the singularity. A different approach [13] is a spectral method, using spherical harmonics, assuming the surface can be mapped to a sphere. This method, applied in [12,29], appears to be advantageous when there are many boundaries that are not greatly deformed. For use with many vesicles, the authors of [29] report difficulties in keeping the method of [7, 32] accurate, perhaps because of the cut-off function needed for the special patch near the singularity. Careful integral methods have the promise of calculating integrals very accurately even for complicated surfaces lacking smoothness; see [6, 15, 17, 27]. An alternative approach, the kernel-free boundary integral method [34, 35], replaces the calculation of the integral by the solution of an interface problem on a regular grid. This approach has the advantage that explicit knowledge of the integral kernel is not needed, and thus it can be applied to more general partial differential equations.

One advantage of the present method is its simplicity. Detailed information about the surface is not required. No special treatment is needed near the singularity, except for the corrections which are added after the summation, using analytical formulas. By design, the errors are uniform with respect to the location; the additional work needed for points close to the boundary is small compared to that for points on the boundary or far away. For an integral with sources on several boundaries, the evaluation at a point on one surface might lead to a nearly singular case, since it could require integration over another surface which is close to the first. Results in [33] showed that the twodimensional version [3] of the present method works well in such cases. 
In this paper we treat single and double layer potentials for harmonic functions. The approach can be extended to integrals for Stokes flow as in [28]; see also [9, 10, 21]. This integration method could be applied to problems with moving interfaces. A discretization of the evolving surface must be chosen and updated. This is often done with representative marker points and triangulation. An alternative might be to use the level set method [22, 26], representing the surface as the zero set of a function whose values are transported by a computed velocity. The velocity would be needed at nearby grid points, in order to update the level set function, rather than on the surface, and the present integration method is designed to be suitable for this purpose.

In Section 2 the quadrature rule for surface integrals is explained. In Section 3 the formulas for calculating single and double layer potentials are given, including the simplified version for points on the surface. A brief discussion of the error estimates is included. Numerical examples illustrating the method with a variety of surfaces are presented in Section 4 . Finally, some possible improvements for this method are discussed briefly in Section 5. The code that produced the examples is available on request from the first two authors.

\section{The quadrature method for surface integrals}

In this section we describe the computational method for integrals on implicitly defined closed surfaces in three space dimensions. The method is also applicable to closed curves in $\mathbb{R}^{2}$ and to hypersurfaces in higher dimensions. Detailed proofs and extensive examples are given in Wilson [30]. Our purpose is to evaluate the surface integral

$$
I=\int_{\Gamma} f(\mathbf{y}) d S_{\mathbf{y}}
$$

We assume $\Gamma$ is a $C^{2 m+1}$ surface, $m \geq 1$, and, for now, $f \in C^{2 m}(\Gamma)$. The method exploits the spectral convergence of the trapezoidal rule without the need for the user to generate a set of overlapping coordinate patches and associated partition of unity. Rather than covering $\Gamma$ with overlapping rectangular patches, we cover $\Gamma$ with certain overlapping surface sets. We define the subsets

$$
\Gamma_{i}=\left\{\mathbf{x} \in \Gamma:\left|\mathbf{n}(\mathbf{x}) \cdot \mathbf{e}_{i}\right|>0\right\}, \quad i=1,2,3
$$

where $\mathbf{n}$ is the unit outward normal at $\mathbf{x}$ and $\left\{\mathbf{e}_{i}\right\}_{i=1}^{3}$ is the standard basis for $\mathbb{R}^{3}$. Thus $\Gamma_{i}$ contains all points in $\Gamma$ where $\mathbf{n}$ is not orthogonal to $\mathbf{e}_{i}$.

The integration method uses a high order, patch-independent quadrature formula for integrals with integrands that vanish outside of a compact subset of one $\Gamma_{i}$. To handle general integrands $f$, we introduce a partition of unity to find functions $\left\{f^{i}\right\}_{i=1}^{3}$ such that $f(\mathbf{x})=\sum_{i=1}^{3} f^{i}(\mathbf{x})$ for all $\mathbf{x} \in \Gamma$ and $f^{i}$ vanishes outside a compact subset of $\Gamma_{i}$. We first design a universal partition of unity on the unit sphere, $S=\left\{\mathbf{u} \in \mathbb{R}^{3}:|\mathbf{u}|=1\right\}$. We start with the smooth bump function defined as $b(r)=e^{r^{2} /\left(r^{2}-1\right)}$ for $|r|<1$ and $b(r)=0$ otherwise. 
Next we choose a fixed angle $\theta$ with $\cos ^{-1}(1 / \sqrt{3})<\theta<\pi / 2$. For each $i \in\{1,2,3\}$ and $\mathbf{u} \in S$ we define

$$
w^{i}(\mathbf{u})=\cos ^{-1}\left(\left|\mathbf{u} \cdot \mathbf{e}_{i}\right|\right), \quad \sigma^{i, \theta}(\mathbf{u})=\frac{b\left(w^{i}(\mathbf{u}) / \theta\right)}{\sum_{j=1}^{3} b\left(w^{j}(\mathbf{u}) / \theta\right)}
$$

Because $\theta>\cos ^{-1}(1 / \sqrt{3})$, the sum is always positive. Furthermore

1. For each $i=1,2,3$, we have $\sigma^{i, \theta} \in C^{\infty}(S)$;

2. For all $\mathbf{u} \in S$, we have $\sum_{i=1}^{3} \sigma^{i, \theta}(\mathbf{u})=1$;

3. For each $i=1,2,3$, the function $\sigma^{i, \theta}$ vanishes outside the compact subset

$$
S_{i, \theta}=\left\{\mathbf{u} \in S:\left|\mathbf{u} \cdot \mathbf{e}_{i}\right| \geq \cos \theta\right\}
$$

To make use of the above partition of unity on the sphere for a general surface $\Gamma$, we apply it to the unit normal $\mathbf{n}(\mathbf{x}) \in C^{2 m}(\Gamma)$. The composition functions $\zeta^{i, \theta}=\sigma^{i, \theta} \circ \mathbf{n}$ on $\Gamma$ satisfy

1. For each $i=1,2,3$, we have $\zeta^{i, \theta} \in C^{2 m}(\Gamma)$;

2. For all $\mathbf{x} \in \Gamma$, we have $\sum_{i=1}^{3} \zeta^{i, \theta}(\mathbf{x})=1$;

3. For each $i=1,2,3$, the function $\zeta^{i, \theta}$ vanishes outside the compact subset of $\Gamma_{i}$ given by

$$
\Gamma_{i, \theta}=\left\{x \in \Gamma:\left|\mathbf{n}(\mathbf{x}) \cdot \mathbf{e}_{i}\right| \geq \cos \theta\right\}
$$

Using the partition of unity, we obtain the exact formula

$$
\int_{\Gamma} f(\mathbf{y}) d S_{\mathbf{y}}=\sum_{i=1}^{3} \int_{\Gamma_{i}} \zeta^{i, \theta}(\mathbf{y}) f(\mathbf{y}) d S_{\mathbf{y}}
$$

where the integrand $f^{i}(\mathbf{y})=\zeta^{i, \theta}(\mathbf{y}) f(\mathbf{y})$ vanishes outside the compact subset $\Gamma_{i, \theta}$ of $\Gamma_{i}$. Finally, the surface integral (2.1) can be approximated by the numerical quadrature

$$
I_{h}=h^{2} \sum_{i=1}^{3} \sum_{\mathbf{x} \in R_{h, i, \theta}} \frac{\zeta^{i, \theta}(\mathbf{x}) f(\mathbf{x})}{\left|\mathbf{n}(\mathbf{x}) \cdot \mathbf{e}_{i}\right|}=h^{2} \sum_{i=1}^{3} \sum_{\mathbf{x} \in R_{h, i, \theta}} \frac{\sigma^{i, \theta}(\mathbf{n}(\mathbf{x})) f(\mathbf{x})}{\left|\mathbf{n}(\mathbf{x}) \cdot \mathbf{e}_{i}\right|}
$$

where

$$
R_{h, i, \theta}=\left\{\mathbf{x} \in \Gamma:\left|\mathbf{n}(\mathbf{x}) \cdot \mathbf{e}_{i}\right| \geq \cos \theta \text { and } p^{i}(\mathbf{x}) \in h \mathbb{Z}^{2}\right\}
$$

and $p^{i}: \mathbb{R}^{3} \mapsto \mathbb{R}^{2}$ is the projection function defined by $p^{1}(\mathbf{x})=\left(x_{2}, x_{3}\right), p^{2}(\mathbf{x})=\left(x_{1}, x_{3}\right)$, $p^{3}(\mathbf{x})=\left(x_{1}, x_{2}\right) . \quad R_{h, i, \theta}$ consists of those points in $\Gamma_{i, \theta}$ that project to grid points in the corresponding plane. The weights $1 /\left|\mathbf{n}(\mathbf{x}) \cdot \mathbf{e}_{i}\right|$ correspond to the area elements of the inverse projections. 
It is proved in [30] that $I_{h}-I=O\left(h^{2 m}\right)$, i.e., the quadrature rule (2.4) is high order accurate, provided the surface $\Gamma$ is $C^{2 m+1}$ and $f$ is $C^{2 m}, m \geq 1$; see Lemma 1, Theorem 2, pp. 9-10, and Lemma 9, Theorem 10, pp. 25-27. In effect the trapezoidal rule applies on coordinate patches covering each $\Gamma_{i, \theta}$. The spacing $h$ must be small enough to resolve the surface. Assume $\Gamma$ is defined as the set $\phi=0$ for some function $\phi$ on an open subset of $\mathbb{R}^{3}$. If $C_{1}=\min |\nabla \phi|$ and $C_{2}=\max \left\|D^{2} \phi\right\|$ near the surface, we need $h<h_{0} \equiv 2 C_{1} \cos \theta / C_{2}$. Thus if the curvature is large, $h$ must be small. The method works if $\phi$ is known only at grid points; see p. 30 of [30].

The use of this rule requires finding the points in $R_{h, i, \theta}$. Given a grid point $\hat{x}$ in a coordinate plane, there may be several points in $\Gamma$ which project to $\hat{x}$, but they are well separated because of the normal condition in (2.2). Consequently, as shown in [30], pp. 11-18, a simple line search algorithm can be used to locate the quadrature points if $h<h_{0}$ : Briefly, to find points in $R_{h, 3, \theta}$, for each $\left(j_{1}, j_{2}\right)$ and $j_{3}$, check whether $\phi$ has a root $\mathbf{x}=$ $\left(j_{1} h, j_{2} h, x_{3}\right)$ with $j_{3} h \leq x_{3} \leq\left(j_{3}+1\right) h$. If so, find a root. If the root is in $R_{h, 3, \theta}$, it is unique. If it is not in $R_{h, 3, \theta}$, reject it. In either case go to the next $j_{3}$. The validity of this algorithm for $h<h_{0}$ is proved in [30].

\section{Evaluation of the layer potentials}

We describe the procedure for computing a single or double layer potential at an arbitrary point, the most difficult case being a location off the surface but close by. For the case of a point on the surface we give more special versions with improved accuracy. Finally we discuss error estimates.

\subsection{The single layer potential}

The single layer potential on $\Gamma$ determined by a density function $\psi$ is

$$
v(\mathbf{x})=\int_{\Gamma} G(\mathbf{y}-\mathbf{x}) \psi(\mathbf{y}) d S_{\mathbf{y}}
$$

where $G$ is the fundamental solution for the Laplacian, $G(x)=-1 / 4 \pi|\mathbf{x}|$. We suppose $\Gamma$ is the boundary of a bounded domain $\Omega$. To evaluate $v$ for $\mathbf{x}$ close to $\Gamma$, we replace $G$ with a smoothed, or regularized, version

$$
G_{\delta}(\mathbf{y})=G(\mathbf{y}) \operatorname{erf}(|\mathbf{y}| / \delta)=-\frac{\operatorname{erf}(|\mathbf{y}| / \delta)}{4 \pi|\mathbf{y}|}
$$

where erf is the error function and $G_{\delta}(\mathbf{0})=-\pi^{-3 / 2}(2 \delta)^{-1}$. The resulting error in the integral is $O(\delta)$. Typically $1 \leq \delta / h \leq 2$. We first compute the regularized integral

$$
v_{\delta}(\mathbf{x})=\int_{\Gamma} G_{\delta}(\mathbf{y}-\mathbf{x}) \psi(\mathbf{y}) d S_{\mathbf{y}}
$$


using the method of Sec. 2. The value obtained is not close to $v(\mathbf{x})$ because of the near singularity. We add corrections for the regularization and discretization to improve the accuracy. Other regularizations could be used, but (3.2) has the advantages that it is simple, $G_{\delta}-G$ decays rapidly in the far field, and manageable formulas can be found for the corrections described below.

To obtain the corrections we first find $\mathbf{z} \in \Gamma$, the closest point on the surface to $\mathbf{x}$, and set $\mathbf{x}=\mathbf{z}+b \mathbf{n}$. Here $\mathbf{n}$ is the unit outward normal to $\Gamma$ at $\mathbf{z} ; b<0$ if $\mathbf{x} \in \Omega$ and $b>0$ if $\mathbf{x} \in \Omega^{c}$ (the complement of the closure of $\Omega$ in $\mathbb{R}^{3}$ ). The correction for regularization of the single layer potential is

$$
\mathcal{T}_{1}=\frac{\delta}{2}(1+H \lambda \delta) \psi(\mathbf{z})\left[|\lambda| \operatorname{erfc}|\lambda|-\frac{e^{-\lambda^{2}}}{\sqrt{\pi}}\right] .
$$

Here $\operatorname{erfc}(r)=1-\operatorname{erf}(r), \lambda=b / \delta$ and $H$ is the mean curvature at $\mathbf{z}, H=\left(\kappa_{1}+\kappa_{2}\right) / 2$, where $\kappa_{1}$ and $\kappa_{2}$ are the principal curvatures. Formulas for computing needed geometric quantities such as $H$ are given in Appendix B, and the sign convention for $H$ is explained.

The discretization correction is a rapidly convergent infinite sum resulting from the Poisson summation formula, applied in each $\Gamma_{i}, i=1,2,3$. Let

$$
E(p, q)=e^{2 p q} \operatorname{erfc}(p+q)+e^{-2 p q} \operatorname{erfc}(-p+q) .
$$

Also let $Q=\left\{n=\left(n_{1}, n_{2}\right) \in \mathbb{Z}^{2}: n_{2}>0\right.$ or $\left(n_{2}=0\right.$ and $\left.\left.n_{1}>0\right)\right\}$. Now suppose $\mathbf{z}$ lies in a system of coordinates, say $\alpha=\left(\alpha_{1}, \alpha_{2}\right)$; in our case, $\mathbf{z} \in \Gamma_{k, \theta}$ for one or more of $k=1,2,3$, and the coordinates at $\mathbf{z}$ are $p^{k}(\mathbf{z}) \in \mathbb{R}^{2}$. Let $g^{i j}$ be the inverse metric tensor at $\mathbf{z}$, and for $n \in Q$, define $\|n\|^{2}=\sum_{i, j} g^{i j} n_{i} n_{j}$. Also write the coordinates of $\mathbf{z}$ as $\left(\alpha_{1}, \alpha_{2}\right)=\left(m_{1}, m_{2}\right) h+\left(v_{1}, v_{2}\right) h$ where $m_{1}, m_{2}$ are integers and $0 \leq v_{1}, v_{2}<1$. Here $\left(v_{1}, v_{2}\right)=v^{(k)}$ and $\|n\|$ depend on the choice of $k$. The discretization correction is

$$
\mathcal{T}_{2}=\frac{h}{4 \pi} \psi(\mathbf{z}) \sum_{k=1}^{3} \sum_{n \in Q} \zeta^{k, \theta}(\mathbf{z}) \cos \left(2 \pi n \cdot v^{(k)}\right) \frac{1}{\|n\|} E(\lambda, \pi \delta\|n\| / h)
$$

with $\zeta^{k, \theta}$ as in Sec. 2. Finally, the computed value $v$ of the integral (3.1) is $v \approx v_{\delta}+\mathcal{T}_{1}+\mathcal{T}_{2}$, where $v_{\delta}$ is the value of (3.3) obtained by the quadrature rule.

\subsection{The double layer potential}

The double layer potential has the form

$$
w(\mathbf{x})=\int_{\Gamma} \frac{\partial G(\mathbf{y}-\mathbf{x})}{\partial \mathbf{n}_{\mathbf{y}}} \varphi(\mathbf{y}) d S_{\mathbf{y}}
$$

It is discontinuous at $\Gamma$. If $\mathbf{x}$ is close to $\Gamma$, we find the closest point $\mathbf{z}$ and distance $b$ as before. We use Green's identities to reduce the singularity and then regularize the kernel, obtaining

$$
w_{\delta}(\mathbf{x})=\int_{\Gamma} \frac{\partial G_{\delta}(\mathbf{y}-\mathbf{x})}{\partial \mathbf{n}_{\mathbf{y}}}[\varphi(\mathbf{y})-\varphi(\mathbf{z})] d S_{\mathbf{y}}+\chi \varphi(\mathbf{z})
$$


Here $\chi=1$ for $x \in \Omega, \chi=0$ on $\Omega^{c}, \chi=\frac{1}{2}$ on $\Gamma$. To form $\partial G_{\delta} / \partial \mathbf{n}$ we use the gradient of the smooth function $G_{\delta}$ introduced in $(3.2)$,

$$
\nabla G_{\delta}(\mathbf{y})=\nabla G(\mathbf{y}) s(|\mathbf{y}| / \delta)=\frac{\mathbf{y}}{4 \pi|\mathbf{y}|^{3}} s(\mathbf{y} \mid / \delta)
$$

with

$$
s(r)=\operatorname{erf}(r)-\frac{2}{\sqrt{\pi}} r e^{-r^{2}}
$$

We compute the integral in 3.8 as in Sec. 2 and again add corrections. The regularization correction for 3.8 is

$$
\mathcal{N}_{1}=\delta^{2}\left(\triangle_{S} \varphi\right) \frac{\lambda}{4}\left[|\lambda| \operatorname{erfc}|\lambda|-\frac{e^{-\lambda^{2}}}{\sqrt{\pi}}\right] .
$$

Here $\triangle_{S} \varphi$ is the surface Laplacian of $\varphi$ at $\mathbf{z}$, which is expressed in coordinates as

$$
\triangle_{S} \varphi=\frac{1}{\sqrt{g}} \sum_{i, j=1}^{2} \frac{\partial}{\partial \alpha_{j}}\left(\sqrt{g} g^{i j} \frac{\partial \varphi}{\partial \alpha_{i}}\right) .
$$

with $g=\operatorname{det} g_{i j}$. The discretization correction is similar to $\mathcal{T}_{2}$ but involves $\partial \varphi / \partial \alpha_{r}, r=1,2$, the coordinate derivatives of $\varphi$ evaluated at $\mathbf{z}$. In our case, on $\Gamma_{k, \theta},\left(\alpha_{1}, \alpha_{2}\right)$ are the two components of $\mathbf{x}=\left(x_{1}, x_{2}, x_{3}\right)$ other than $x_{k}$, and we write these derivatives as $\partial_{r}^{(k)} \varphi(\mathbf{z})$. The correction is

$$
\mathcal{N}_{2}=-\frac{\delta \lambda}{2} \sum_{k=1}^{3} \sum_{r=1}^{2} c_{r}^{(k)} \zeta^{k, \theta}(\mathbf{z}) \partial_{r}^{(k)} \varphi(\mathbf{z})
$$

where

$$
c_{r}^{(k)}=\sum_{n \in Q} \sum_{s=1}^{2} \sin \left(2 \pi n \cdot v^{(k)}\right) \frac{g^{r s} n_{s}}{\|n\|} E(\lambda, \pi \delta\|n\| / h)
$$

with $\|n\|$ as before. The computed value of (3.7) is $w \approx w_{\delta}+\mathcal{N}_{1}+\mathcal{N}_{2}$.

\subsection{The potentials evaluated on the surface}

We now treat the important special case of evaluation at a point $\mathbf{x}$ on the surface $\Gamma$. For this case, in contrast to the nearly singular case, it is not difficult to modify the regularized kernels to have higher accuracy by imposing moment conditions. Thus no corrections are needed for regularization. The method is easier to use than in the general case, and the error is typically smaller, as seen in the examples in Section 4. A strategy for producing these improved kernels from the ones already chosen in (3.2) and (3.9) is described in [2] for the single layer and in [4] for the double layer. For $G_{\delta}$ as defined in (3.2), the error in regularization, i.e. the difference between the integrals in $(3.3)$ and $(3.1)$, is $O(\delta)$. For the new version of $G_{\delta}$ the error is $O\left(\delta^{5}\right)$ for the special case of evaluation on the surface, and similarly for the double layer. 
To evaluate the single layer potential (3.1) at $x \in \Gamma$, we use the new version of $G_{\delta}$ with $O\left(\delta^{5}\right)$ accuracy,

$$
G_{\delta}(\mathbf{y})=-\frac{s(|\mathbf{y}| / \delta)}{4 \pi|\mathbf{y}|}, \quad s(r)=\operatorname{erf}(r)+\frac{2}{3 \sqrt{\pi}}\left(5 r-2 r^{3}\right) e^{-r^{2}}
$$

and $G_{\delta}(\mathbf{0})=-(4 / 3) \pi^{-3 / 2} \delta^{-1}$. In place of the corrections 3.4 . 3.6) we have $\mathcal{T}_{1}=0$ and

$$
\mathcal{T}_{2}=\frac{\delta}{\pi} \psi(\mathbf{z}) \sum_{k=1}^{3} \sum_{n \in Q} \zeta^{k, \theta}(\mathbf{z}) \cos \left(2 \pi n \cdot v^{(k)}\right) F(\xi)
$$

where, with $\xi=2 \pi\|n\| \delta / h$ and $\|n\|$ as before,

$$
F(\xi)=\frac{\pi}{\xi} \operatorname{erfc}(\xi / 2)+\pi^{1 / 2} \frac{\delta}{h} e^{-\xi^{2} / 4}\left(1+\frac{\xi^{2}}{6}\right)
$$

The derivation of (3.14) is similar to that in Sec. 2 of [2] for an $O\left(\delta^{3}\right)$ version, with the formula (3.15) corresponding to (3.28), (2.23) in [4].

To evaluate the double layer potential (3.7) at $\mathbf{x} \in \Gamma$, we use 3.8 with $\chi=\frac{1}{2}$ and $\nabla G_{\delta}$ of the form (3.9) but with (3.10) replaced by

$$
s(r)=\operatorname{erf}(r)-\frac{2}{\sqrt{\pi}}\left(r-\frac{2 r^{3}}{3}\right) e^{-r^{2}} .
$$

In this case no corrections are needed, that is, $\mathcal{N}_{1}=\mathcal{N}_{2}=0$. Formula (3.17) was derived in [4], p. 607.

\subsection{Error analysis}

For the general case of points close to $\Gamma$, the corrections $\mathcal{N}_{1}, N_{2}$ for the double layer were derived in [4]. Those for the single layer can be found similarly; we include a brief derivation of $\mathcal{T}_{1}$ in Appendix A. The discretization corrections are based on the Poisson Summation Formula. After applying both corrections to either the single or double layer potential, the remaining error has the form (cf. Theorem 1.2 in [4])

$$
\epsilon \leq C_{1} \delta^{3}+C_{2} h^{2} e^{-c_{0}(\delta / h)^{2}}
$$

as $\delta, h \rightarrow 0$, assuming $\delta / h$ is bounded below, with $C_{1}, C_{2}$ depending on derivatives of the surface and density functions. The two terms represent the regularization error and discretization error, respectively. The constant $c_{0}$ is determined by the choice of local coordinate system. It is important for accuracy that $c_{0}$ does not become small, so that the sums in the discretization corrections converge rapidly, and so that the second error term in (3.18) is comparable to the first in practice. It was shown in [4], Sec. 3, that the estimate 3.18 holds provided

$$
c_{0}<\pi^{2} \gamma^{2}, \quad \gamma^{2} \equiv \min _{|k|=1} \sum_{i, j} g^{i j} k_{i} k_{j}
$$


(A factor $1 / 2$ in $c_{0}$ in [4] was arbitrary.) Here $g^{i j}=\left(g_{i j}\right)^{-1}$ is the inverse metric tensor. With coordinates $\left(\alpha_{1}, \alpha_{2}\right), g_{i j}=T_{i} \cdot T_{j}$, where $T_{j}=\partial \mathbf{x} / \partial \alpha_{j}, j=1,2$ are the tangent vectors. If the coordinate system distorts distances significantly, $\gamma$ could be small, and thus the accuracy of the method depends on the choice of coordinates.

In our case, the coordinate systems are those determined by the projections. For $\Gamma_{3}$, the coordinates are $x_{1}, x_{2}$, with $x_{3}=f\left(x_{1}, x_{2}\right)$, and similarly for $\Gamma_{1}, \Gamma_{2}$. From the expression for $g^{i j}$ in Appendix B, it is not difficult to see that in this case $\gamma=\left(1+f_{1}^{2}+f_{2}^{2}\right)^{-1 / 2}=\left|\mathbf{n} \cdot \mathbf{e}_{3}\right| \geq$ $\cos \theta$ in $\Gamma_{3, \theta}$, where $\theta$ is the angle chosen in Sec. 2. Thus (3.18) holds with $c_{0} \approx \pi^{2} \cos ^{2} \theta$, and the exponential in (3.18) can be made quite small. For example, if $\delta=2 h$ and $\theta=60^{\circ}$, the exponential is .00005; if $\delta=2.5 h$ and $\theta=70^{\circ}$ it is .0007. In practice the accuracy is about $O\left(h^{3}\right)$ for usual values of $h$, with proper choice of parameters. Alternatively, we could take $\delta=\mathrm{Ch}^{q}$ for any $q<1$ and thereby obtain convergence as $h \rightarrow 0$ with order $O\left(h^{3 q}\right)$.

For the sums in (3.6), (3.13) we only need a few terms because of the rapid decay as $n$ increases. In the corrections we may evaluate $H$ and $\triangle_{S} \varphi$ not at the closest point $\mathbf{z}$ but rather at a neighboring grid point, using formulas in Appendix B. The $O(h)$ errors in these quantities do not change the order of accuracy of the corrections. Similarly, in the discretization corrections, the $g^{i j}$ and $\partial_{r}^{(k)} \varphi$ only need to be computed within $O(h)$.

For the special case with $\mathbf{x} \in \Gamma$, the first term in the error estimate (3.18) improves to $\delta^{5}$. (See Theorem 1.1 of [4] for the double layer.) Thus we can take $\delta / h$ somewhat larger to reduce the discretization error in the second term. If $\delta=C h^{q}, q<1$, then the error is $O\left(h^{5 q}\right)$ as $q \rightarrow 0$.

\section{Numerical Results}

This section presents examples evaluating the sum of a double layer and single layer potential on five different surfaces,

$$
u(\mathbf{x})=w(\mathbf{x})+v(\mathbf{x})=\int_{\Gamma} \frac{\partial G(\mathbf{y}, \mathbf{x})}{\partial \mathbf{n}_{\mathbf{y}}} \varphi(\mathbf{y}) d s_{\mathbf{y}}-\int_{\Gamma} G(\mathbf{y}, \mathbf{x}) \psi(\mathbf{y}) d s_{\mathbf{y}}
$$

In all the examples the potential $u$ is chosen to be

$$
u(x, y, z)=\left\{\begin{array}{ll}
(\sin x+\sin y) e^{z} & \text { if }(x, y, z) \in \Omega \\
0 & \text { if }(x, y, z) \in \Omega^{c}
\end{array} .\right.
$$

The densities $\varphi$ and $\psi$ are determined by the jumps in $u$ and $\partial u / \partial n$. The integrals are calculated given these densities, and the result is compared with the exact $u$. This choice of test problem allows us to have an exact solution with an arbitrary surface.

In each example, the domain $\Omega$ is embedded into a cubic box $\mathcal{B}=(-L, L)^{3}$ with $L=1.1$. The box is partitioned into a uniform grid $\mathcal{T}_{h}$ with mesh parameter $h=2 L / N$, the width of a grid cell. We call a grid node irregular if the stencil of the second-order Laplacian $\Delta_{h}$ crosses the boundary $\Gamma$; otherwise it is regular. 
The numerical values of $u(\mathbf{x})=w(\mathbf{x})+v(\mathbf{x})$ are first computed at the irregular grid nodes, as well as the neighboring nodes in their stencil, using the procedure of Sec. 3 . The sums $w_{\delta}, v_{\delta}$ for the smoothed potentials are found and the corrections are added. The summation is done using a slight modification of the treecode algorithm of Duan and Krasny [11], which was designed for use with Ewald summation. (The kernel in their code has a factor of erfc rather than erf, and their solutions are periodic rather than in free space.) In the treecode we chose the degree of Taylor polynomials $p=12$, the separation parameter $s=.5$, and the capacity, or maximum number of points in a leaf, $N_{0}=20$.

Having calculated $u(\mathbf{x})$ at grid nodes $\mathbf{x}$ near $\Gamma$, we can now find values at all the regular grid nodes of $\mathcal{T}_{h}$ by inverting the discrete Laplacian, using a procedure suggested in [20]. Let $u_{h, \delta}$ denote the value of $u$ already computed at the nodes close to $\Gamma$ as nearly singular integrals. We formulate a Poisson problem for an approximation $u_{h}$ on $\mathcal{T}_{h}$ to the exact $u$,

$$
\Delta_{h} u_{h}=\left\{\begin{array}{ll}
\Delta_{h} u_{h, \delta} & \text { at irregular grid nodes } \\
0 & \text { at regular grid nodes }
\end{array} .\right.
$$

We solve for $u_{h}$ with a fast Poisson solver on the box $\mathcal{B}$ with zero boundary condition. At the regular nodes, the truncation error is $O\left(h^{2}\right)$, since the exact solution is a smooth, harmonic function away from $\Gamma$. For the irregular nodes, $u_{h, \delta}-u$ is about $O\left(h^{3}\right)$, so that $\Delta_{h} u_{h, \delta}-\Delta_{h} u=O\left(h^{3} / h^{2}\right)=O(h)$. In summary, $\Delta_{h}\left(u_{h}-u\right)$ is $O\left(h^{2}\right)$ at regular nodes and $O(h)$ at irregular nodes. Despite the first order truncation error near $\Gamma$, the resulting $u_{h}$ is second order accurate, i.e., $u_{h}-u=O\left(h^{2}\right)$, uniformly on $\mathcal{T}_{h}$, a fact proved in [5]. While this method is efficient, more accurate values could be computed as integrals or otherwise.

We also computed the values of $u(\mathbf{x})$ at grid nodes $\mathbf{x}$ on $\Gamma$ using the method in Section 3.3. (The exact value is the average of the inside and outside limits.) In this case we used direct summation to provide an unambiguous test of the accuracy.

For Example 1, the surface $\Gamma$ is an ellipsoid given by

$$
\frac{x^{2}}{a^{2}}+\frac{y^{2}}{b^{2}}+\frac{z^{2}}{c^{2}}=1
$$

with $a=1, b=.8, c=.6$, and rotated by an orthogonal matrix to test the effect of grid alignment. Example 2 is a thinner ellipsoid, with $a=1, b=c=.4$, without rotation. Example 3 is a torus

$$
\left(\sqrt{x^{2}+y^{2}}-c\right)^{2}+z^{2}=a^{2}
$$

with $a=.3$ and $c=.7$. Example 4 is a molecular surface with four atoms, similar to one of the definitions in [8],

$$
\sum_{k=1}^{4} \exp \left(\left|\mathbf{x}-\mathbf{x}_{k}\right|^{2} / r^{2}\right)=c
$$




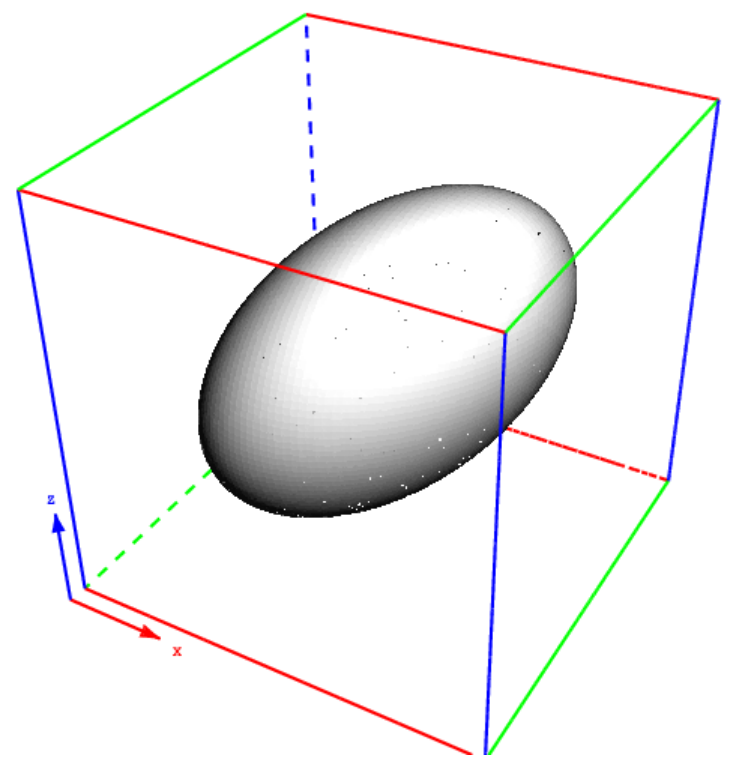

Figure 1: The rotated $(1, .8 . .6)$ ellipsoid

Here the centers are $(\sqrt{3} / 3,0,-\sqrt{6} / 12),(-\sqrt{3} / 6, .5,-\sqrt{6} / 12),(-\sqrt{3} / 6,-.5,-\sqrt{6} / 12)$, $(0,0, \sqrt{6} / 4)$ and $r=.5, c=.6$. Example 5 is a surface obtained by revolving a Cassini oval,

$$
\left(x^{2}+y^{2}+z^{2}+a^{2}\right)^{2}-4 a^{2}\left(x^{2}+y^{2}\right)=b^{4}
$$

with $a=.65$ and $b=.7$.

The errors for each example are presented in the tables with $N=64,128$ or 256 and with $\delta / h=1,2$, or 3 . Both $L^{2}$ and maximum, or $L^{\infty}$, errors are given. They are displayed first for the irregular grid points, then for the regular grid points, and finally for the quadrature nodes on the surface. In the $L^{2}$ norms, points are given equal weight, and thus for the quadrature nodes this measure effectively gives extra weight to the overlap regions. In all the examples the angle $\theta$ in the partition of unity on the sphere is $70^{\circ}$. The final table gives the number of quadrature nodes on each surface with $N=256$.

For the irregular points the smallest errors are generally for $\delta / h=1$, while accuracy approaching $O\left(h^{3}\right)$ is observable with $\delta / h=2$ or 3 . (We have found that errors are larger with $\delta / h<1$.) The errors at the regular points is $O\left(h^{2}\right)$ as expected. The errors at the quadrature points are generally smaller than those at the irregular points for $\delta / h=2$ or 3 , but not for $\delta / h=1$; this reflects the fact that the method of Section 3.3 for evaluation on the surface improves the smoothing error directly but the discretization error is improved indirectly by the smoothing. With $\delta / h=3$ the errors on the surface decrease rapidly with refinement. We repeated the computations on the surface with angle $60^{\circ}$ and found similar but slightly larger errors. To test further refinement, we computed $u$ on the surface for Example 1 with $N=512$. With angle $70^{\circ}$ and $\delta / h=2$, the $L^{2}$ and $L^{\infty}$ errors were 3.03E-8 


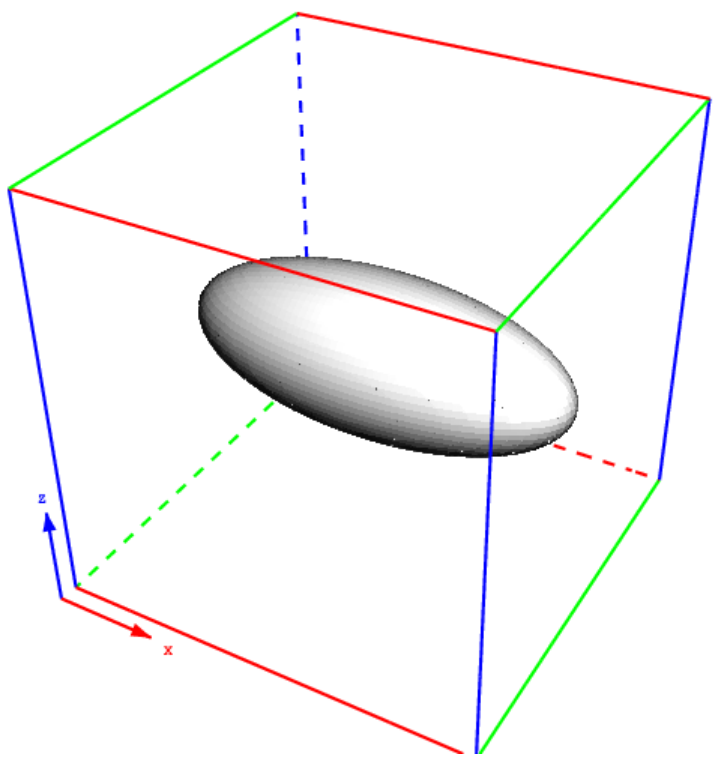

Figure 2: The $(1, .4, .4)$ ellipsoid

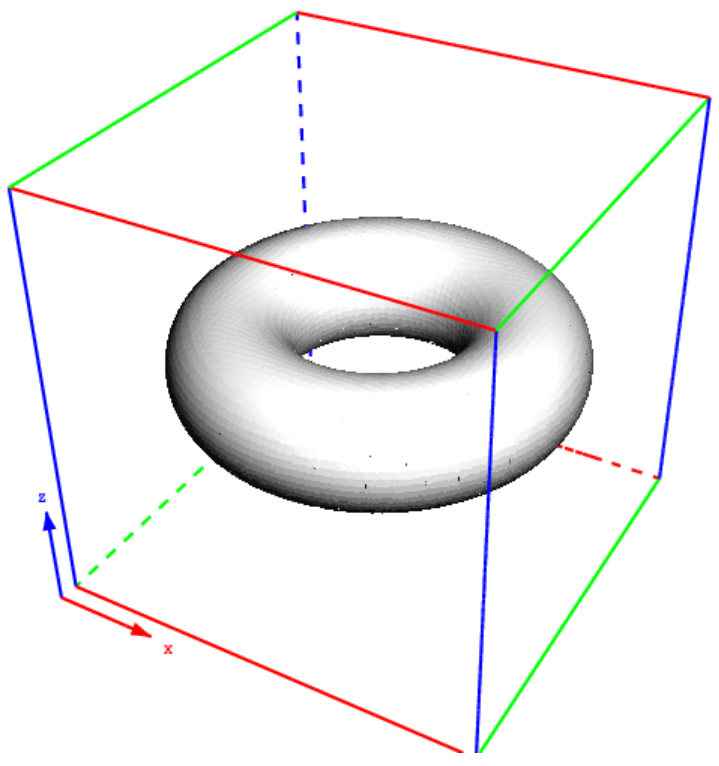

Figure 3: The torus 


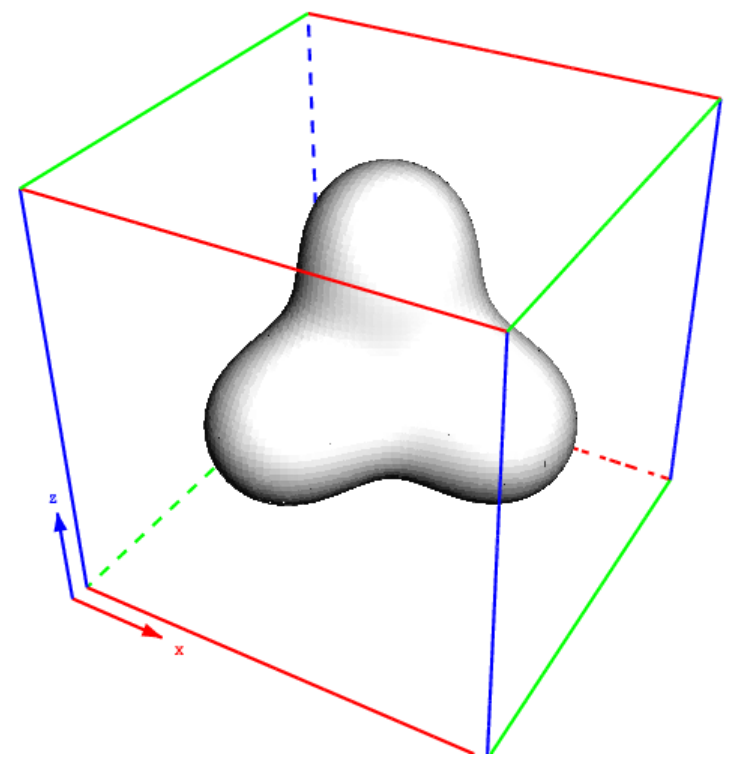

Figure 4: The four-atom molecular surface

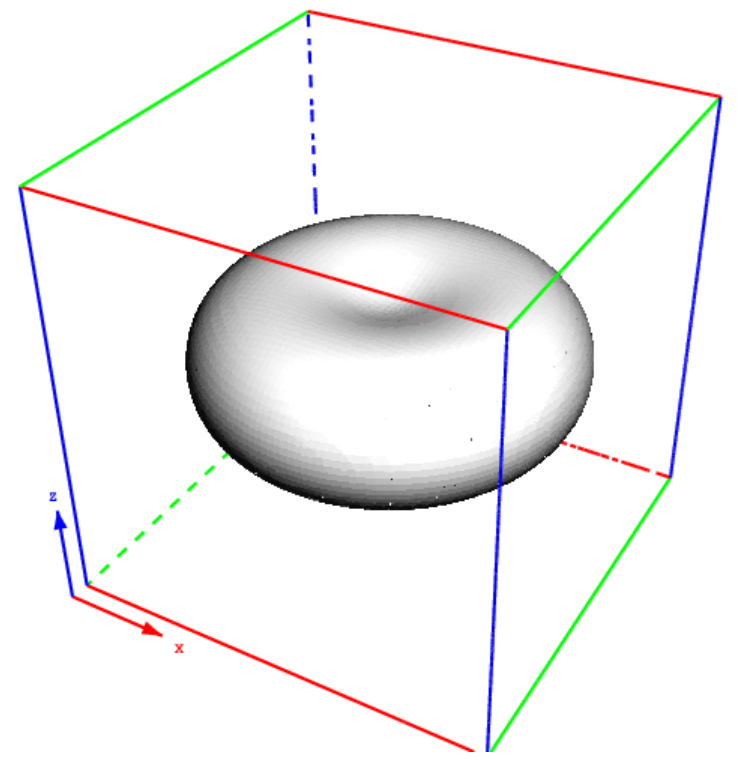

Figure 5: The Cassini oval surface 
and 7.43E-7; with $\delta / h=3$ they were 3.09E-10 and 1.33E-8. With angle $60^{\circ}$, they were 6.83E$10,3.82 \mathrm{E}-8$ for $\delta / h=2$ and $2.42 \mathrm{E}-10,1.09 \mathrm{E}-8$ for $\delta / h=3$. We conclude that for practical use we can reliably choose $\delta / h=2$ for values at points near the surface and $\delta / h=3$ with the special method of Sec. 3.3 for points on the surface.

In these calculations we used analytical values of $g^{i j}$ since the surface was specified. However these could easily be computed from grid values of a level set function (see Appendix B) and are needed only to $O(h)$ accuracy. We used analytical values of the densities $\varphi$ and $\psi$ at the quadrature points and computed other values numerically from these. We did so because in solving an integral equation we would only know values at the quadrature points. Occasionally the computation of $\partial_{r}^{(k)} \varphi$ in 3.12 failed for lack of nearby quadrature points near the edge of the support of one $\zeta^{k, \theta}$. In such a case we set the contribution to zero since $\zeta^{k, \theta}$ is very small there. We also treated the ellipsoid of Example 1 without rotation; the errors were similar to those displayed with the rotation but slightly smaller. We tried an ellipsoid thinner than in Example 2; we found that the interpolation stencil needed for the corrections $\mathcal{T}_{1}, \mathcal{N}_{1}$ failed with $N=64$ but worked with larger $N$.

Table 1: Errors for the rotated $(1, .8, .6)$ ellipsoid. In each table the angle $\theta$ is $70^{\circ}$ and $h=2.2 / N$ for the $N^{3}$ grid. $L^{2}$ and $L^{\infty}$ errors are displayed for (i) irregular grid points near the surface, (ii) regular grid points, and (iii) quadrature points on the surface.

\begin{tabular}{|c|c|c|c|c|c|c|c|}
\hline$\delta$ & grid & $\left\|e_{h}^{\text {irreg }}\right\|_{2}$ & $\left\|e_{h}^{\text {irreg }}\right\|_{\infty}$ & $\left\|e_{h}^{\text {reg }}\right\|_{2}$ & $\left\|e_{h}^{\text {reg }}\right\|_{\infty}$ & $\left\|e_{h}^{\text {quad }}\right\|_{2}$ & $\left\|e_{h}^{\text {quad }}\right\|_{\infty}$ \\
\hline \hline \multirow{3}{*}{$h$} & $64^{3}$ & $3.32 \mathrm{E}-5$ & $2.57 \mathrm{E}-4$ & $3.29 \mathrm{E}-5$ & $5.05 \mathrm{E}-4$ & $9.80 \mathrm{E}-5$ & $1.24 \mathrm{E}-3$ \\
\cline { 2 - 8 } & $128^{3}$ & $4.14 \mathrm{E}-6$ & $3.54 \mathrm{E}-5$ & $7.96 \mathrm{E}-6$ & $1.07 \mathrm{E}-4$ & $2.10 \mathrm{E}-5$ & $3.33 \mathrm{E}-4$ \\
\cline { 2 - 8 } & $256^{3}$ & $9.91 \mathrm{E}-7$ & $6.55 \mathrm{E}-6$ & $2.10 \mathrm{E}-6$ & $2.92 \mathrm{E}-5$ & $5.36 \mathrm{E}-6$ & $8.69 \mathrm{E}-5$ \\
\hline \hline \multirow{3}{*}{$2 h$} & $64^{3}$ & $1.39 \mathrm{E}-4$ & $8.64 \mathrm{E}-4$ & $1.82 \mathrm{E}-4$ & $2.39 \mathrm{E}-3$ & $3.15 \mathrm{E}-5$ & $4.32 \mathrm{E}-4$ \\
\cline { 2 - 8 } & $128^{3}$ & $1.78 \mathrm{E}-5$ & $1.14 \mathrm{E}-4$ & $4.35 \mathrm{E}-5$ & $4.67 \mathrm{E}-4$ & $1.71 \mathrm{E}-6$ & $3.94 \mathrm{E}-5$ \\
\cline { 2 - 8 } & $256^{3}$ & $3.33 \mathrm{E}-6$ & $1.39 \mathrm{E}-5$ & $1.13 \mathrm{E}-5$ & $1.36 \mathrm{E}-4$ & $1.38 \mathrm{E}-7$ & $4.69 \mathrm{E}-6$ \\
\hline \hline \multirow{3}{*}{$3 h$} & $64^{3}$ & $4.78 \mathrm{E}-4$ & $2.91 \mathrm{E}-3$ & $3.65 \mathrm{E}-4$ & $5.06 \mathrm{E}-3$ & $2.59 \mathrm{E}-5$ & $2.83 \mathrm{E}-4$ \\
\cline { 2 - 8 } & $128^{3}$ & $5.75 \mathrm{E}-5$ & $3.82 \mathrm{E}-4$ & $8.79 \mathrm{E}-5$ & $9.45 \mathrm{E}-4$ & $1.13 \mathrm{E}-6$ & $1.68 \mathrm{E}-5$ \\
\cline { 2 - 8 } & $256^{3}$ & $7.84 \mathrm{E}-6$ & $4.78 \mathrm{E}-5$ & $2.27 \mathrm{E}-5$ & $2.70 \mathrm{E}-4$ & $1.67 \mathrm{E}-8$ & $6.36 \mathrm{E}-7$ \\
\hline
\end{tabular}

\section{Discussion}

The numerical results illustrate the performance of the method and are in general agreement with the qualitative predictions. They show that reasonable accuracy can be obtained with moderate resolution, and the observed order of accuracy gives confidence that the errors will reduce with further refinement. Here we comment on possible improvements.

As noted in Section 3, the discretization error depends on the angle $\theta$ in the partition 
Table 2: Errors for the $(1,4, .4)$ ellipsoid

\begin{tabular}{|c|c|c|c|c|c|c|c|}
\hline$\delta$ & grid & $\left\|e_{h}^{\text {irreg }}\right\|_{2}$ & $\left\|e_{h}^{\text {irreg }}\right\|_{\infty}$ & $\left\|e_{h}^{\text {reg }}\right\|_{2}$ & $\left\|e_{h}^{\text {reg }}\right\|_{\infty}$ & $\left\|e_{h}^{\text {quad }}\right\|_{2}$ & $\left\|e_{h}^{\text {quad }}\right\|_{\infty}$ \\
\hline \hline \multirow{3}{*}{$h$} & $64^{3}$ & $4.46 \mathrm{E}-5$ & $3.27 \mathrm{E}-4$ & $2.62 \mathrm{E}-5$ & $4.39 \mathrm{E}-4$ & $1.48 \mathrm{E}-4$ & $9.26 \mathrm{E}-4$ \\
\cline { 2 - 8 } & $128^{3}$ & $8.53 \mathrm{E}-6$ & $8.73 \mathrm{E}-5$ & $6.79 \mathrm{E}-6$ & $1.21 \mathrm{E}-4$ & $3.25 \mathrm{E}-5$ & $3.07 \mathrm{E}-4$ \\
\cline { 2 - 8 } & $256^{3}$ & $1.08 \mathrm{E}-6$ & $1.39 \mathrm{E}-5$ & $1.69 \mathrm{E}-6$ & $3.15 \mathrm{E}-5$ & $7.35 \mathrm{E}-6$ & $6.70 \mathrm{E}-5$ \\
\hline \hline \multirow{3}{*}{$2 h$} & $64^{3}$ & $3.29 \mathrm{E}-4$ & $2.33 \mathrm{E}-3$ & $1.69 \mathrm{E}-4$ & $2.05 \mathrm{E}-3$ & $4.95 \mathrm{E}-5$ & $2.66 \mathrm{E}-4$ \\
\cline { 2 - 8 } & $128^{3}$ & $4.29 \mathrm{E}-5$ & $2.73 \mathrm{E}-4$ & $4.07 \mathrm{E}-5$ & $4.17 \mathrm{E}-4$ & $6.82 \mathrm{E}-6$ & $9.36 \mathrm{E}-5$ \\
\cline { 2 - 8 } & $256^{3}$ & $5.11 \mathrm{E}-6$ & $3.57 \mathrm{E}-5$ & $1.04 \mathrm{E}-5$ & $1.05 \mathrm{E}-4$ & $4.04 \mathrm{E}-7$ & $1.00 \mathrm{E}-5$ \\
\hline \hline \multirow{3}{*}{$3 h$} & $64^{3}$ & $1.12 \mathrm{E}-3$ & $6.85 \mathrm{E}-3$ & $3.37 \mathrm{E}-4$ & $4.29 \mathrm{E}-3$ & $4.59 \mathrm{E}-5$ & $2.35 \mathrm{E}-4$ \\
\cline { 2 - 8 } & $128^{3}$ & $1.43 \mathrm{E}-4$ & $9.62 \mathrm{E}-4$ & $8.07 \mathrm{E}-5$ & $7.85 \mathrm{E}-4$ & $5.22 \mathrm{E}-6$ & $5.20 \mathrm{E}-5$ \\
\cline { 2 - 8 } & $256^{3}$ & $1.76 \mathrm{E}-5$ & $1.23 \mathrm{E}-4$ & $2.09 \mathrm{E}-5$ & $2.22 \mathrm{E}-4$ & $2.03 \mathrm{E}-7$ & $3.91 \mathrm{E}-6$ \\
\hline
\end{tabular}

Table 3: Errors for the torus

\begin{tabular}{|c|c|c|c|c|c|c|c|}
\hline$\delta$ & grid & $\left\|e_{h}^{\text {irreg }}\right\|_{2}$ & $\left\|e_{h}^{\text {irreg }}\right\|_{\infty}$ & $\left\|e_{h}^{\text {reg }}\right\|_{2}$ & $\left\|e_{h}^{\text {reg }}\right\|_{\infty}$ & $\left\|e_{h}^{\text {quad }}\right\|_{2}$ & $\left\|e_{h}^{\text {quad }}\right\|_{\infty}$ \\
\hline \hline \multirow{3}{*}{$h$} & $64^{3}$ & $7.19 \mathrm{E}-5$ & $3.57 \mathrm{E}-4$ & $5.17 \mathrm{E}-5$ & $5.03 \mathrm{E}-4$ & $1.48 \mathrm{E}-4$ & $1.29 \mathrm{E}-3$ \\
\cline { 2 - 8 } & $128^{3}$ & $8.61 \mathrm{E}-6$ & $7.56 \mathrm{E}-5$ & $9.04 \mathrm{E}-6$ & $1.14 \mathrm{E}-4$ & $3.14 \mathrm{E}-5$ & $3.05 \mathrm{E}-4$ \\
\cline { 2 - 8 } & $256^{3}$ & $1.02 \mathrm{E}-6$ & $9.61 \mathrm{E}-6$ & $2.16 \mathrm{E}-6$ & $2.01 \mathrm{E}-5$ & $7.02 \mathrm{E}-6$ & $7.50 \mathrm{E}-5$ \\
\hline \hline \multirow{3}{*}{$2 h$} & $64^{3}$ & $2.42 \mathrm{E}-4$ & $7.94 \mathrm{E}-4$ & $1.74 \mathrm{E}-4$ & $1.42 \mathrm{E}-3$ & $8.08 \mathrm{E}-5$ & $4.16 \mathrm{E}-4$ \\
\cline { 2 - 8 } & $128^{3}$ & $2.89 \mathrm{E}-5$ & $9.54 \mathrm{E}-5$ & $4.41 \mathrm{E}-5$ & $3.31 \mathrm{E}-4$ & $8.53 \mathrm{E}-6$ & $8.34 \mathrm{E}-5$ \\
\cline { 2 - 8 } & $256^{3}$ & $3.52 \mathrm{E}-6$ & $1.25 \mathrm{E}-5$ & $1.12 \mathrm{E}-5$ & $8.85 \mathrm{E}-5$ & $4.76 \mathrm{E}-7$ & $7.46 \mathrm{E}-6$ \\
\hline \hline \multirow{3}{*}{$3 h$} & $64^{3}$ & $8.17 \mathrm{E}-4$ & $2.68 \mathrm{E}-3$ & $3.17 \mathrm{E}-4$ & $3.01 \mathrm{E}-3$ & $6.35 \mathrm{E}-5$ & $2.80 \mathrm{E}-4$ \\
\cline { 2 - 8 } & $128^{3}$ & $9.92 \mathrm{E}-5$ & $3.28 \mathrm{E}-4$ & $8.51 \mathrm{E}-5$ & $6.93 \mathrm{E}-4$ & $7.05 \mathrm{E}-6$ & $4.85 \mathrm{E}-5$ \\
\cline { 2 - 8 } & $256^{3}$ & $1.22 \mathrm{E}-5$ & $4.22 \mathrm{E}-5$ & $2.18 \mathrm{E}-5$ & $1.84 \mathrm{E}-4$ & $2.46 \mathrm{E}-7$ & $2.80 \mathrm{E}-6$ \\
\hline
\end{tabular}

of unity on the unit sphere, defined in Sec. 2. We need $\theta>55^{\circ}$ to cover the sphere. As $\theta$ increases toward $90^{\circ}$ we expect the accuracy to deteriorate because of the dependence of the discretization error on $\theta$, as explained in Sec. 3.4. Here we used $\theta=70^{\circ}$ as a compromise between the extremes. In our experiments the errors were not very sensitive to the choice of angle for $60^{\circ} \leq \theta \leq 80^{\circ}$. We found slightly larger errors with $\theta=60^{\circ}$ than for $70^{\circ}$. A possible explanation is that the gradient of the partition of unity functions is larger for the smaller angle. It is unclear whether this can be improved or is an inherent limitation.

In the discretization corrections $\mathcal{T} 2, \mathcal{N} 2$ we summed over $n=\left(n_{1}, n_{2}\right)$ with $\left|n_{j}\right| \leq$ 20 , but the number of terms actually needed is much smaller. In fact for $\delta / h \geq 2$ these corrections are usually negligible. They could be modified to include an estimate of the number of terms needed to avoid unnecessary work.

The treecode of [11] cannot be used directly with the method of Section 3.3 for evaluation on the surface because of the differences in the regularized kernels. However, the treecode could be applied in this case by modifying the recurrence formulas for the 
Table 4: Errors for the molecular surface

\begin{tabular}{|c|c|c|c|c|c|c|c|}
\hline$\delta$ & grid & $\left\|e_{h}^{\text {irreg }}\right\|_{2}$ & $\left\|e_{h}^{\text {irreg }}\right\|_{\infty}$ & $\left\|e_{h}^{\text {reg }}\right\|_{2}$ & $\left\|e_{h}^{\text {reg }}\right\|_{\infty}$ & $\left\|e_{h}^{\text {quad }}\right\|_{2}$ & $\left\|e_{h}^{\text {quad }}\right\|_{\infty}$ \\
\hline \hline \multirow{4}{*}{$h$} & $64^{3}$ & $6.84 \mathrm{E}-5$ & $4.16 \mathrm{E}-4$ & $4.02 \mathrm{E}-5$ & $4.81 \mathrm{E}-4$ & $1.61 \mathrm{E}-4$ & $1.47 \mathrm{E}-3$ \\
\cline { 2 - 8 } & $128^{3}$ & $5.98 \mathrm{E}-6$ & $5.55 \mathrm{E}-5$ & $1.09 \mathrm{E}-5$ & $1.50 \mathrm{E}-4$ & $3.24 \mathrm{E}-5$ & $3.24 \mathrm{E}-4$ \\
\cline { 2 - 8 } & $256^{3}$ & $1.03 \mathrm{E}-6$ & $1.30 \mathrm{E}-5$ & $2.73 \mathrm{E}-6$ & $3.96 \mathrm{E}-5$ & $7.60 \mathrm{E}-6$ & $8.33 \mathrm{E}-5$ \\
\hline \hline \multirow{3}{*}{$2 h$} & $6^{3}$ & $3.30 \mathrm{E}-4$ & $1.53 \mathrm{E}-3$ & $2.51 \mathrm{E}-4$ & $3.73 \mathrm{E}-3$ & $6.80 \mathrm{E}-5$ & $6.07 \mathrm{E}-4$ \\
\cline { 2 - 8 } & $128^{3}$ & $3.99 \mathrm{E}-5$ & $1.81 \mathrm{E}-4$ & $6.02 \mathrm{E}-5$ & $7.37 \mathrm{E}-4$ & $3.43 \mathrm{E}-6$ & $6.78 \mathrm{E}-5$ \\
\cline { 2 - 8 } & $256^{3}$ & $4.96 \mathrm{E}-6$ & $2.36 \mathrm{E}-5$ & $1.45 \mathrm{E}-5$ & $1.57 \mathrm{E}-4$ & $2.32 \mathrm{E}-7$ & $4.34 \mathrm{E}-6$ \\
\hline \hline \multirow{3}{*}{$3 h$} & $64^{3}$ & $1.11 \mathrm{E}-3$ & $5.12 \mathrm{E}-3$ & $5.08 \mathrm{E}-4$ & $8.56 \mathrm{E}-3$ & $6.35 \mathrm{E}-5$ & $4.35 \mathrm{E}-4$ \\
\cline { 2 - 8 } & $128^{3}$ & $1.38 \mathrm{E}-4$ & $6.26 \mathrm{E}-4$ & $1.18 \mathrm{E}-4$ & $1.58 \mathrm{E}-3$ & $2.01 \mathrm{E}-6$ & $3.46 \mathrm{E}-5$ \\
\cline { 2 - 8 } & $256^{3}$ & $1.72 \mathrm{E}-5$ & $8.17 \mathrm{E}-5$ & $2.80 \mathrm{E}-5$ & $3.27 \mathrm{E}-4$ & $5.40 \mathrm{E}-8$ & $1.24 \mathrm{E}-6$ \\
\hline
\end{tabular}

Table 5: Errors for the Cassini oval surface

\begin{tabular}{|c|c|c|c|c|c|c|c|}
\hline$\delta$ & grid & $\left\|e_{h}^{\text {irreg }}\right\|_{2}$ & $\left\|e_{h}^{\text {irreg }}\right\|_{\infty}$ & $\left\|e_{h}^{\text {reg }}\right\|_{2}$ & $\left\|e_{h}^{\text {reg }}\right\|_{\infty}$ & $\left\|e_{h}^{\text {quad }}\right\|_{2}$ & $\left\|e_{h}^{\text {quad }}\right\|_{\infty}$ \\
\hline \hline \multirow{3}{*}{$h$} & $64^{3}$ & $4.87 \mathrm{E}-5$ & $2.94 \mathrm{E}-4$ & $3.40 \mathrm{E}-5$ & $3.60 \mathrm{E}-4$ & $1.20 \mathrm{E}-4$ & $1.01 \mathrm{E}-3$ \\
\cline { 2 - 8 } & $128^{3}$ & $3.78 \mathrm{E}-6$ & $3.07 \mathrm{E}-5$ & $7.25 \mathrm{E}-6$ & $6.59 \mathrm{E}-5$ & $2.37 \mathrm{E}-5$ & $2.27 \mathrm{E}-4$ \\
\cline { 2 - 8 } & $256^{3}$ & $6.82 \mathrm{E}-7$ & $5.75 \mathrm{E}-6$ & $1.82 \mathrm{E}-6$ & $1.68 \mathrm{E}-5$ & $5.62 \mathrm{E}-6$ & $5.75 \mathrm{E}-5$ \\
\hline \hline \multirow{3}{*}{$2 h$} & $64^{3}$ & $2.02 \mathrm{E}-4$ & $8.64 \mathrm{E}-4$ & $1.55 \mathrm{E}-4$ & $1.20 \mathrm{E}-3$ & $5.18 \mathrm{E}-5$ & $3.53 \mathrm{E}-4$ \\
\cline { 2 - 8 } & $128^{3}$ & $2.46 \mathrm{E}-5$ & $1.20 \mathrm{E}-4$ & $3.80 \mathrm{E}-5$ & $3.38 \mathrm{E}-4$ & $3.15 \mathrm{E}-6$ & $3.76 \mathrm{E}-5$ \\
\cline { 2 - 8 } & $256^{3}$ & $3.10 \mathrm{E}-6$ & $1.56 \mathrm{E}-5$ & $9.59 \mathrm{E}-6$ & $8.17 \mathrm{E}-5$ & $2.19 \mathrm{E}-7$ & $3.63 \mathrm{E}-6$ \\
\hline \hline \multirow{3}{*}{$3 h$} & $64^{3}$ & $6.83 \mathrm{E}-4$ & $2.65 \mathrm{E}-3$ & $2.97 \mathrm{E}-4$ & $2.58 \mathrm{E}-3$ & $4.47 \mathrm{E}-5$ & $2.20 \mathrm{E}-4$ \\
\cline { 2 - 8 } & $128^{3}$ & $8.61 \mathrm{E}-5$ & $3.91 \mathrm{E}-4$ & $7.35 \mathrm{E}-5$ & $7.50 \mathrm{E}-4$ & $1.84 \mathrm{E}-6$ & $1.86 \mathrm{E}-5$ \\
\cline { 2 - 8 } & $256^{3}$ & $1.08 \mathrm{E}-5$ & $5.13 \mathrm{E}-5$ & $1.86 \mathrm{E}-5$ & $1.78 \mathrm{E}-4$ & $4.65 \mathrm{E}-8$ & $6.96 \mathrm{E}-7$ \\
\hline
\end{tabular}

Taylor coefficients as derived in [11]. This could be done in future work.

Other than [11], fast summation methods have not been developed specifically for regularized kernels. Among existing codes, one possible alternative that might be used in the present computations is the kernel-independent fast multipole method (KIFMM) of L. Ying, G. Biros, D. Zorin [31]. We have calculated examples using this code, even though it was not intended for regularized kernels. We found difficulty maintaining good accuracy, especially with larger $\delta / h$, perhaps because the regularization degrades the accuracy of the linear problems solved in the KIFMM. We emphasize that this is a use of the KIFMM for which it was not intended. A summation method of fast multipole type designed particularly for these regularized kernels could improve the efficiency of this method without loss of accuracy. 
Table 6: The number of quadrature nodes with $N=256$

\begin{tabular}{|c|c|c|c|c|c|}
\hline Example & 1 & 2 & 3 & 4 & 5 \\
\hline Number of nodes & 144388 & 70790 & 142168 & 126789 & 133014 \\
\hline
\end{tabular}

\section{Acknowledgments}

Research of the first author was supported in part by the National Science Foundation of the USA under Grant DMS-1312654. Research of the second author was supported in part by the National Natural Science Foundation of China under Grants DMS-11101278 and DMS-91130012. Research of the second author was also supported in part by the Young Thousand Talents Program of China. Research of the third author was supported by the National Science Foundation of the USA under Grant DMS-0806482.

\section{A Regularization error for the single layer potential}

The regularization correction for the single layer potential, evaluated at a point near the surface, can be derived using the method of [4], Sec. 2. With $G_{\delta}$ and $G$ as in (3.2), we approximate the error in the single layer potential with density $\psi$, evaluated at a point $\mathbf{x}$ near the surface. Since the error is local, we write it as an integral in one coordinate patch, regarding $\psi$ as a function of $\alpha=\left(\alpha_{1}, \alpha_{2}\right)$,

$$
\epsilon=\int\left[G_{\delta}(\mathbf{y}(\alpha)-\mathbf{x})-G(\mathbf{y}(\alpha)-\mathbf{x})\right] \psi(\alpha) d S(\alpha) .
$$

For simplicity, we will assume that $\mathbf{x}$ is along the normal line from $0 \in \Gamma$, so that $\mathbf{x}=b \mathbf{n}_{0}$ for some $b$, where $\mathbf{n}_{0}$ is the unit normal at 0 . We also assume the coordinates are chosen so that $\alpha(0)=0, g_{i j}(\alpha)=\delta_{i j}+O\left(|\alpha|^{2}\right)$, and the tangent vectors $T_{1}, T_{2}$ have the directions of principal curvature. Thus

$$
\epsilon=\frac{1}{4 \pi} \int \frac{\operatorname{erfc}(r / \delta)}{r} \psi(\alpha) d S(\alpha), \quad r=|y(\alpha)-x|
$$

Proceeding as before, we make a near-identity coordinate change $\alpha \rightarrow \xi$ such that $|\xi|^{2}+b^{2}=r^{2}$. We get

$$
\epsilon=\frac{1}{4 \pi} \int \frac{\operatorname{erfc}\left(\sqrt{|\xi|^{2}+b^{2}} / \delta\right)}{\left(|\xi|^{2}+b^{2}\right)^{1 / 2}} w(\xi, b) d \xi
$$

with

$$
w(\xi, b)=\psi\left|\frac{\partial \alpha}{\partial \xi}\right|\left|T_{1} \times T_{2}\right|
$$

We will see that we can neglect terms in $w$ of the form $O\left(|\xi|^{2}+b^{2}\right)$. We can approximate $\psi$ in $\xi$, with leading term $\psi_{0}=\psi(0)$,

$$
\psi=\psi_{0}+\psi_{j}(1+b q / 2) \xi_{j}+\frac{1}{2} \psi_{i j} \xi_{i} \xi_{j}+O\left(|\xi|^{3}\right)+O\left(b^{3}\right) .
$$


where $q=\kappa_{1} \xi_{1}^{2} /|\xi|^{2}+\kappa_{2} \xi_{2}^{2} /|\xi|^{2}$ and $\kappa_{1}, \kappa_{2}$ are the principal curvatures at 0 . For the other two factors in $w$, we have

$$
\operatorname{det}(\partial \alpha / \partial \xi)=1+b q+O\left(|\xi|^{2}\right)+O\left(b^{2}\right) .
$$

and

$$
\left|T_{1} \times T_{2}\right|=1+O\left(|\xi|^{2}\right) .
$$

In the $\xi$-integral for $\epsilon$, the odd part of $w$ will contribute zero. Thus we can replace $w$ with an approximation to its even part. Combining the three factors above, we get

$$
w^{e v e n}(\xi, b)=\psi_{0}(1+b q)+O\left(|\xi|^{2}+b^{2}\right)
$$

We now substitute in the integral, change to polar coordinates, and substitute $|\xi|=\delta$ s and $b=\delta \lambda$ to obtain

$$
\epsilon=\frac{\delta}{2} \psi_{0}(1+\delta \lambda H) \int_{0}^{\infty} \frac{\operatorname{erfc}\left(\sqrt{s^{2}+\lambda^{2}}\right)}{\left(s^{2}+\lambda^{2}\right)^{1 / 2}} s d s+O\left(\delta^{3}\right)
$$

with $H=\left(\kappa_{1}+\kappa_{2}\right) / 2$, the mean curvature. With $r=\sqrt{s^{2}+\lambda^{2}}$, and $s d s=r d r$, the integral simplifies to

$$
I=\int_{0}^{\infty} \frac{\operatorname{erfc}(r)}{r} s d s=\int_{|\lambda|}^{\infty} \operatorname{erfc}(r) d r=e^{-\lambda^{2}} / \sqrt{\pi}-|\lambda| \operatorname{erfc}|\lambda|
$$

and finally

$$
\epsilon=\frac{\delta}{2} \psi_{0}(1+\delta \lambda H)\left(e^{-\lambda^{2}} / \sqrt{\pi}-|\lambda| \operatorname{erfc}|\lambda|\right)+O\left(\delta^{3}\right)
$$

leading to the correction (3.4).

\section{B Formulas for Monge Patches}

We summarize formulas needed for the corrections of Sec. 3 when applied in a coordinate system such as $x_{3}=f\left(x_{1}, x_{2}\right)$, often called a Monge patch. Given $f$, let $f_{i}=\partial f / \partial x_{i}, i=1,2$, and similarly for a second derivative $f_{i j}$. The metric tensor $\left(g_{i j}\right)$ and its inverse $\left(g^{i j}\right)$ are

$$
\left(g_{i j}\right)=\left(\begin{array}{cc}
1+f_{1}^{2} & f_{1} f_{2} \\
f_{1} f_{2} & 1+f_{2}^{2}
\end{array}\right), \quad\left(g^{i j}\right)=\frac{1}{g}\left(\begin{array}{cc}
1+f_{2}^{2} & -f_{1} f_{2} \\
-f_{1} f_{2} & 1+f_{1}^{2}
\end{array}\right) .
$$

where $g=\operatorname{det}\left(g_{i j}\right)=1+f_{1}^{2}+f_{2}^{2}$. The Gauss curvature is

$$
K=g^{-2}\left(f_{11} f_{22}-f_{12}^{2}\right)
$$

The mean curvature is

$$
H= \pm \frac{1}{2} g^{-3 / 2}\left[\left(1+f_{2}^{2}\right) f_{11}+\left(1+f_{1}^{2}\right) f_{22}-2 f_{1} f_{2} f_{12}\right]
$$


where the sign is + if $x_{3}>f\left(x_{1}, x_{2}\right)$ outside and - otherwise.

The surface Laplacian has the general formula

$$
\triangle_{S} \varphi=\sum_{i, j} \frac{1}{\sqrt{g}} \partial_{j}\left(\sqrt{g} g^{i j} \partial_{i} \varphi\right)=\sum_{i, j} g^{i j}\left(\partial_{i} \partial_{j} \varphi\right)+\sum_{i} c_{i} \partial_{i} \varphi
$$

where

$$
c_{i}=\sum_{j} \frac{1}{\sqrt{g}} \partial_{j}\left(\sqrt{g} g^{i j}\right)=\sum_{j} \frac{1}{\sqrt{g}} \partial_{j}\left(\frac{1}{\sqrt{g}}\left(g g^{i j}\right)\right)
$$

With some calculation we find

$$
\frac{1}{\sqrt{g}} \partial_{j}\left(\frac{1}{\sqrt{g}}\right)=-g^{-2}\left(f_{1} f_{1 j}+f_{2} f_{2 j}\right)
$$

and subsequently

$$
c_{i}=g^{-2} f_{i}\left[2 f_{1} f_{2} f_{12}-\left(1+f_{2}^{2}\right) f_{11}-\left(1+f_{1}^{2}\right) f_{22}\right]=\mp \frac{2}{\sqrt{g}} H f_{i}
$$

Suppose the surface is defined by $\phi\left(x_{1}, x_{2}, x_{3}\right)=0$, with $\phi>0$ outside. Near a given point, there is at least one Monge patch; suppose we can solve for $x_{3}=f\left(x_{1}, x_{2}\right)$ as above. By differentiating implicitly we get $f_{i}=-\phi_{i} / \phi_{3}$ etc. We can use these to express $g_{i j}$ and $g^{i j}$. A more convenient expression for the mean curvature $H$ on a surface $\{\phi=0\}$ is based on the classical formula

$$
2 H=-\nabla \cdot n=-\nabla \cdot(\nabla \phi /|\nabla \phi|)
$$

If we carry out the differentiation we get

$$
2 H=-|\nabla \phi|^{-3}\left(\phi_{i i} \phi_{j}^{2}-\phi_{i} \phi_{j} \phi_{i j}\right)
$$

summed over $i, j$. After canceling and combining terms, we obtain a formula such as in [22], p. 12.

\section{References}

[1] K. E. Atkinson, The Numerical Solution of Integral Equations of the Second Kind, Cambridge University Press, 1997.

[2] J. T. Beale, A convergent boundary integral method for three-dimensional water waves, Math. Comp., 70 (2001), 977-1029.

[3] J. T. Beale and M. C. Lai, A method for computing nearly singular integrals, SIAM J. Numer. Anal., 38 (2001), 1902-1925.

[4] J. T. Beale, A grid-based boundary integral method for elliptic problems in three-dimensions, SIAM J. Numer. Anal., 42 (2004), 599-620.

[5] J. T. Beale and A. T. Layton, On the accuracy of finite difference methods for elliptic problems with interfaces, Commun. Appl. Math. Comput. Sci., 1 (2006), 91-119. 
[6] J. Bremer and Z. Gimbutas, A Nyström method for weakly singular integral operators on surfaces, J. Comput. Phys., 231 (2012), 4885-4903.

[7] O. Bruno and L. Kunyansky, A fast, high-order algorithm for the solution of surface scattering problems: Basic implementation, tests, and applications, J. Comput. Phys., 169 (2001), $80-110$.

[8] M. Chen and B. Lu, TMSmesh: A robust method for molecular surface mesh generation using a trace technique, J. Chem. Theory Comput., 7 (2011), 203-12.

[9] R. Cortez, The method of regularized Stokeslets, SIAM J. Sci. Comput., 23 (2001), 1204-25.

[10] R. Cortez, L. Fauci and A. Medovikov, The method of regularized Stokeslets in three dimensions: analysis, validation, and application to helical swimming, Phys. Fluids, 17 (2005), $1-14$.

[11] Z.-H. Duan and R. Krasny, An Ewald summation based multipole method, J. Chem. Phys. $113,(2000), 3492-5$.

[12] M. Ganesh and I. G. Graham, A high-order algorithm for obstacle scattering in three dimensions, J. Comput. Phys., 198 (2004), 211-42.

[13] I. G. Graham and I. H. Sloan, Fully discrete spectral boundary integral methods for Helmholtz problems on smooth closed surfaces in $\mathbb{R}^{3}$, Numerische Mathematik, 92 (2002), 289-323.

[14] W. Hackbusch, Integral Equations, Theory and Numerical Treatment, Birkhäuser, 1995.

[15] J. Helsing and R. Ojala, On the evaluation of layer potentials close to their sources, J. Comput. Phys., 227 (2008), 2899-2921.

[16] J. Helsing, A higher-order singularity subtraction technique for the discretization of singular integral operators on curved surfaces, preprint, 2013.

[17] A. Klöckner, A. Barnett, L. Greengard and M. O'Neil, Quadrature by expansion: A new method for the evaluation of layer potentials, J. Comput. Phys., 252 (2013), 332-349.

[18] K. Lindsay and R. Krasny, A particle method and adaptive treecode for vortex sheet motion in three-dimensional flow, J. Comput. Phys., 172 (2001), 879-907.

[19] O. Marin, O. Runborg and A.-K. Tornberg, Corrected trapezoidal rules for a class of singular functions, IMA J. Numer. Anal., 34 (2014), 1509-1540.

[20] A. Mayo, Fast high order accurate solution of Laplace's equation on irregular regions, SIAM J. Sc. Statist. Comput., 6 (1985), 144-157.

[21] H.-N. Nguyen and R. Cortez, Reduction of the regularization error of the method of regularized Stokeslets for a rigid object immersed in a three-dimensional Stokes flow, Commun. Comput. Phys., 15 (2014), 126-152.

[22] S. Osher and R. Fedkiw, Level Set Methods and Dynamic Implicit Surfaces, Springer, 2003.

[23] C. Pozrikidis, Interfacial dynamics for Stokes flow, J. Comput. Phys., 169 (2001), 250-301.

[24] C. Pozrikidis, A Practical Guide to Boundary Element Methods with the Software Library BEMLIB, C.R.C., 2002.

[25] S. Sauter and C. Schwab, Boundary Element Methods, Springer, 2010.

[26] J. Sethian, Level Set Methods and Fast Marching Methods, Cambridge Univ. Press, 1998.

[27] M. Taus, G. Rodin and T. J. R. Hughes Isogeometric analysis of boundary integral equations, Math. Models Methods Appl. Sci. 26 (2016), 1447-80.

[28] S. Tlupova and J. T. Beale, Nearly singular integrals in 3d Stokes flow, Commun. Comput. Phys., 14 (2013), 1207-27.

[29] S. K. Veerapaneni, A. Rahimian, G. Biros and D. Zorin, A fast algorithm for simulating vesicle flows in three dimensions, J. Comput. Phys., 230 (2011), 5610-34.

[30] J. R. Wilson, On computing smooth, singular and nearly singular integrals on implicitly 
defined surfaces, Ph.D. thesis, Duke University (2010),

http://search.proquest. com/docview/744476497

[31] L. Ying, G. Biros and D. Zorin, A kernel-independent adaptive fast multipole algorithm in two and three dimensions, J. Comput. Phys., 196 (2004), 591-626.

[32] L. Ying, G. Biros and D. Zorin, A high-order 3d boundary integral equation solver for elliptic pdes in smooth domains, J. Comput. Phys., 219 (2006), 247-75.

[33] W.-J. Ying and J. T. Beale, A fast accurate boundary integral method for potentials on closely packed cells, Commun. Comput. Phys., 14 (2013), 1073-93.

[34] W.-J. Ying and W.-C. Wang, A kernel-free boundary integral method for implicitly defined surfaces, J. Comput. Phys., 252 (2013), 606-624.

[35] W.-J. Ying and W.-C. Wang, A kernel-free boundary integral method for variable coefficients elliptic PDEs, Commun. Comput. Phys., 15 (2014), 1108-1140.

[36] A. Z. Zinchenko, M. A. Rother and R. H. Davis, Cusping, capture, and breakup of interacting drops by a curvatureless boundary-integral algorithm, J. Fluid Mech., 391 (1999), 249-92. 